\title{
Ensemble seasonal forecast of extreme water inflow into a large reservoir
}

\author{
A. N. Gelfan ${ }^{1,2}$, Yu. G. Motovilov ${ }^{1,2}$, and V. M. Moreido ${ }^{1}$ \\ ${ }^{1}$ Water Problems Institute of RAS, Moscow, Russia \\ ${ }^{2}$ P.P. Shirshov Institute of Oceanology of RAS, Moscow, Russia \\ Correspondence to: V. M. Moreido (moreido@mail.ru)
}

Received: 15 April 2015 - Accepted: 15 April 2015 - Published: 11 June 2015

\begin{abstract}
An approach to seasonal ensemble forecast of unregulated water inflow into a large reservoir was developed. The approach is founded on a physically-based semi-distributed hydrological model ECOMAG driven by Monte-Carlo generated ensembles of weather scenarios for a specified lead-time of the forecast ( 3 months ahead in this study). Case study was carried out for the Cheboksary reservoir (catchment area is $374000 \mathrm{~km}^{2}$ ) located on the middle Volga River. Initial watershed conditions on the forecast date (1 March for spring freshet and 1 June for summer low-water period) were simulated by the hydrological model forced by daily meteorological observations several months prior to the forecast date. A spatially distributed stochastic weather generator was used to produce time-series of daily weather scenarios for the forecast lead-time. Ensemble of daily water inflow into the reservoir was obtained by driving the ECOMAG model with the generated weather time-series. The proposed ensemble forecast technique was verified on the basis of the hindcast simulations for 29 spring and summer seasons beginning from 1982 (the year of the reservoir filling to capacity) to 2010. The verification criteria were used in order to evaluate an ability of the proposed technique to forecast freshet/low-water events of the pre-assigned severity categories.
\end{abstract}

\section{Introduction}

Providing a seasonal streamflow forecast is crucial for largescale water reservoirs operation, as water management plans are designed for several months ahead in order to optimize the efficiency of water supply infrastructure and the operation of hydro-electric plants, to mitigate against the effects of flooding, and to meet other requirements related to the efficient use of water resources and flood protection systems. However, current levels of accuracy of seasonal forecasts of spring freshet and summer low-water hydrological characteristics are far from meeting society's needs (Luo et al., 2007). For the last decade, there has been a gradual trend from deterministic regression-based methodology (still served as a basis for long-term operational forecasting for water supply management around the globe) towards ensemble, hydrological model-based methodology aimed to increase informational content of forecasts and, hopefully, to improve the forecast accuracy (see overviews of Cloke and Pappenberger,
2009; Gelfan and Motovilov, 2009, among others). Ensemble forecasts provide not only an estimate of the most likely future state of a hydrological system, but also yield a range of possible outcomes that allow quantitative estimating of forecast uncertainty and destroying "the illusion of certainty in a user's mind" (Krzysztofowicz, 2001). In the area of seasonal hydrological forecasting, this methodology usually combines a hydrological model with ensembles of future weather scenarios for a specified lead time, which are then used as inputs for the model. Commonly, the weather scenarios are taken from the historical observations which assumed to be representative of possible future meteorological conditions (NWS Ensemble Streamflow Prediction System (Connelly et al., 1999), Volga-Kama Water Resources Prediction System (Gelfan and Motovilov, 2009)). Hydrological ensemble predicted on the basis of meteorological observations includes, at best, several dozen members. Such a small ensemble can cause difficulties with the ability of the forecasting method to provide estimates of the magnitude and probability of oc- 


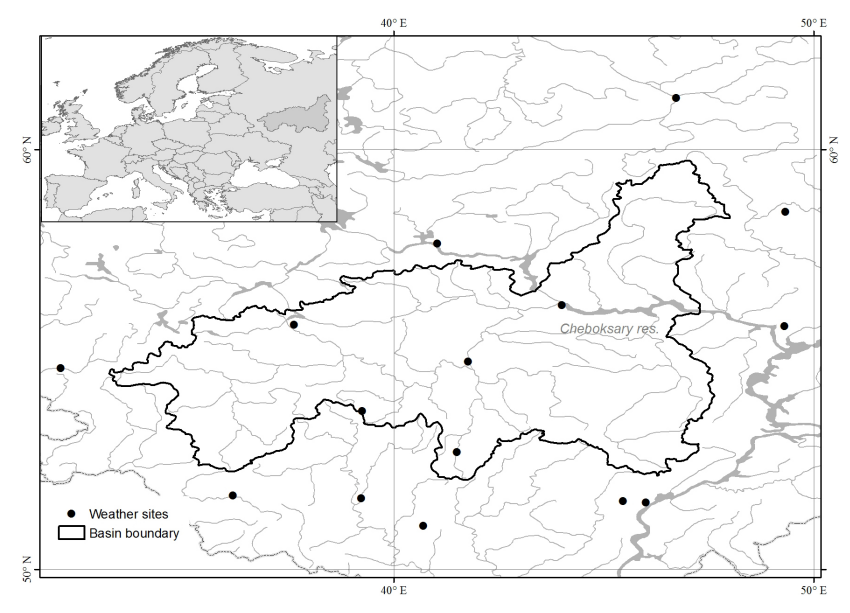

Figure 1. Case study: Cheboksary reservoir watershed.

currence of possible extreme hydrological events. This problem can be overcome combining hydrological model with a stochastic weather generator as proposed by Kuchment and Gelfan (2007). A hydrological model that is forced by synthetic weather data allows reproducing responses of a hydrological system with respect to many possible meteorological impacts, including those that may be unobserved for the lead time considered, but physically possible.

In this study, we applied this approach to seasonal ensemble forecast of unregulated water inflow into a large reservoir during both spring freshet and summer low-water periods. The proposed forecasting methodology is based on the combined use of the physically-based semi-distributed model ECOMAG (ECOlogical Model for Applied Geophysics; Motovilov et al., 1999a) and stochastic weather generator SFRWG (Spatial-Fragment based Weather Generator; Gelfan, Motovilov, 2014). Case study was carried out for the Cheboksary reservoir, filled to capacity in 1982 to become the 11th member of Volga-Kama reservoir cascade. It receives regulated inflow from the Nizhegorodskoe reservoir as well as unregulated flow from its main trubutaries Oka, Sura and Vetluga rivers. The watershed area of unregulated water inflow into Cheboksary reservoir is $373800 \mathrm{~km}^{2}$ (Fig. 1). Water regime of the study basin is characterized by spring high-water freshet followed by summer low-water periods.

\section{Hydrological model and stochastic weather generator: brief description and results of application to the study basin}

The ECOMAG model describes processes of snow accumulation and melt, soil freezing and thawing, water infiltration into unfrozen and frozen soil, evapotranspiration, thermal and water regime of soil, overland, subsurface and channel flow. The ECOMAG model utilizes semi-distributed approach with the whole river basin interpreted as a number

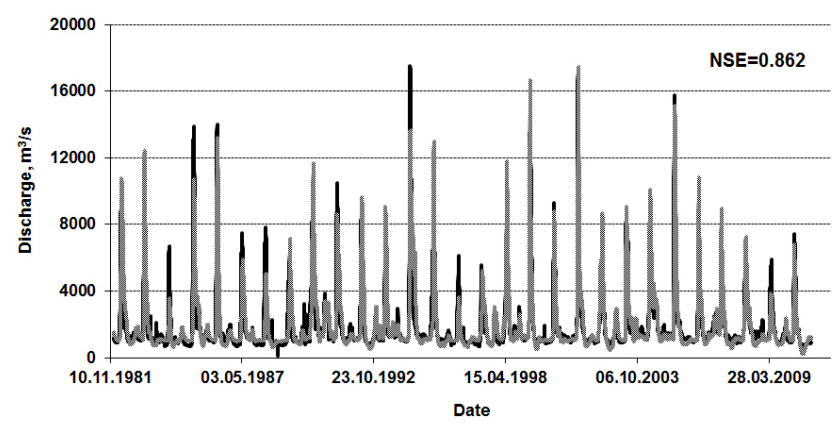

Figure 2. Observed (black line) and simulated (gray line) hydrographs of unregulated lateral water inflow into the Cheboksary reservoir.

of sub-basins. It takes into consideration topography, soil and land cover characteristics of the particular sub-basin. For each sub-basin hydraulic properties of soil as well as landcover properties are scaled taking into account sub-basin area. Most of the parameters are physically meaningful and can be assigned from literature or derived through available measured characteristics of topography, soil, and land-cover. Some key-parameters are calibrated against streamflow measurements and, if available, measurements of the internal basin variables (snow characteristics, soil moisture, groundwater level, etc.). The ECOMAG model is forced by daily time series of air temperature, air humidity and precipitation. Examples of the model application for hydrological prediction in many river basins of very different size and locations are presented, particularly, by Motovilov et al. (1999b), Motovilov and Gelfan (2013), and Gelfan et al. (2014).

The ECOMAG model was calibrated and validated against the Cheboksary reservoir daily water inflow observations beginning from 1 January 1982 (the 1st year after the reservoir filling to capacity) to 31 December 2010: calibration covered the period of 1982-1999, the rest of the data were used for the model validation. Spatially distributed daily meteorological input was assigned from measurements at 15 weather sites (Fig. 1). Figure 2 compares observed with simulated hydrographs of daily inflow into the reservoir during the whole period and demonstrated good performance of simulation (the overall Nash-and-Sutcliffe efficiency criterion for daily discharge simulations is 0.86 ). Figure 3 demonstrates ability of the model to reproduce cumulative water inflow during spring freshet and summer low-water seasons.

The spatially distributed stochastic weather generator SFRWG (Gelfan and Motovilov, 2014) is a set of stochastic models that use existing weather records to produce long time-series of synthetic daily weather variables, which statistical properties, both spatial and temporal, are expected to be similar to those of the actual data. The generator includes stochastic models of daily precipitation, air temperature and air humidity deficit and is based on the method of spatial fragments. The latter presents a modification of the known 

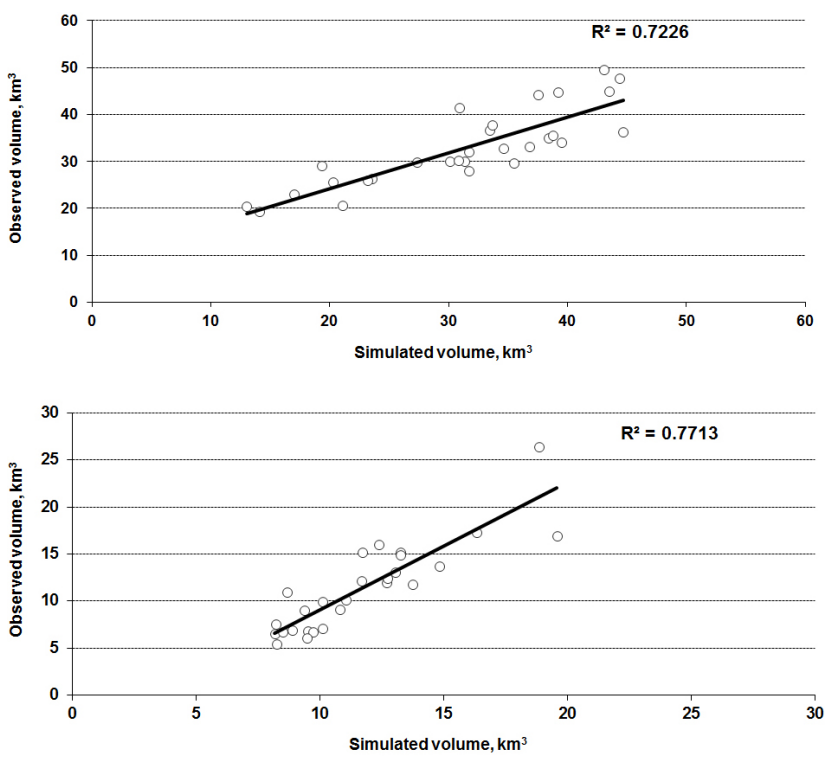

Figure 3. Observed and simulated volumes of seasonal inflow into the Cheboksary reservoir (top - spring freshet season; bottom summer low-water season).

method of fragments proposed by Svanidze (1980) for modeling time-series of high autocorrelation. The SFRWG generator contains only seven parameters characterizing spatial patterns (fragments) of the simulated variables. The parameters were derived from the available meteorological observations during 60-year period (1953-2012) at 15 gauges located within the study basin (Fig. 1). Statistics of the multiyear Monte-Carlo generated time-series of daily meteorological variables were compared with the corresponding statistics derived from observations. As an example, Fig. 4 demonstrates spatial correlations of the generated and observed variables. Detailed results of the SFRWG evaluation are presented in Gelfan and Motovilov (2014).

Thus, one can see from the above illustrations that both the hydrological model ECOMAG and the weather generator SFRWG satisfactory passes the testing procedures and we have combined these tools for providing ensemble forecast of water inflow into the Cheboksary reservoir.

\section{Ensemble forecast and its verification: description of the used techniques}

The proposed technique of the ensemble forecast of seasonal water inflow into the Cheboksary reservoir includes the following procedures: (1) spinup ECOMAG-based simulation ("warm start") using meteorological station observations several months prior to the forecast date in order to calculate the nowcast of the initial watershed hydrological state (soil, snow and channel water contents, groundwater level, soil freezing depth, etc.) that initializes the forecast; (2) SFRWG-based generation of 500-member ensemble of spatially distributed daily weather scenarios for the forecast lead-time (three months); (3) obtaining ensemble of daily water inflow into the reservoir by driving the ECOMAG with ensemble of generated weather scenarios beginning on day of the forecast initialization and extending for three months.

Verification of the proposed forecasting technique was carried out on the basis of hindcast simulations for 29 spring and 29 summer seasons, from 1982 (reservoir filling year) to 2010. The forecast initialization date for the spring freshet season was 1 March (the lead-time period is 1 March31 May), the corresponding date for summer low-water seasonal forecast was 1 June (the lead-time period is 1 June31 August). For each season, the forecasted hydrological event indicators were derived from the simulated daily hydrograph ensembles and applied for the forecast performance evaluation. The event indicator $\mathrm{EI}_{i j}$ for $i$ th season $(i=1, \ldots$, $\left.N_{Y}=29\right)$ and $j$ th ensemble member $\left(j=1, \ldots, N_{E}=500\right)$ was calculated as the normalized runoff volume by formula

$\mathrm{EI}_{i j}=\frac{W_{i j}-M_{W}}{S_{W}}$

where $W_{i j}$ is the runoff volume predicted by the ECOMAG driven by $j$ th weather scenario for $i$ th $\quad$ season; $\quad M_{W}=\frac{1}{N_{Y} N_{E}} \sum_{i=1}^{N_{Y}} \sum_{j=1}^{N_{E}} W_{i j} \quad$ and $\quad S_{W}=$ $\sqrt{\frac{1}{\left(N_{Y} N_{E}-1\right)} \sum_{i=1}^{N_{Y}} \sum_{j=1}^{N_{E}}\left(W_{i j}-M_{W}\right)^{2}}$ are the overall mean and standard deviation of runoff volume, respectively.

Freshet-event indicator, $\mathrm{FI}_{i j}$, and low-water-event indicator, $\mathrm{LWI}_{i j}$, were calculated using Eq. (1), where runoff volume $W_{i}^{j}$ was determined for spring and summer seasons, respectively. States of spring freshet and summer low-water events were determined in the same manner as, for instance, for meteorological drought event (e.g. Standardised Precipitation Index; McKee et al., 1993) or for hydrological drought event (e.g. Streamflow Drought Index; Nalbantis, 2008). Five states of the hydrological event were considered (Table 1).

The above event indicators, obtained from the ensemble of simulated hydrographs were compared with identical event indicators that were derived from the measured daily hydrographs for 29 seasons of observations. The forecast verification methods widely used in meteorological forecasting practice (see, for instance, Wilks, 1995) were applied.

\section{Results}

The results of the ensemble forecast are summarized in the multi-category contingency Table 2 . It shows frequencies of observations and respective forecasts in the various event categories presented in Table 1. Diagonal elements of the table denote frequencies of the forecasted events of the specific category that meet the observed events in the same category. A perfect forecast has elements of non-zero values only along 

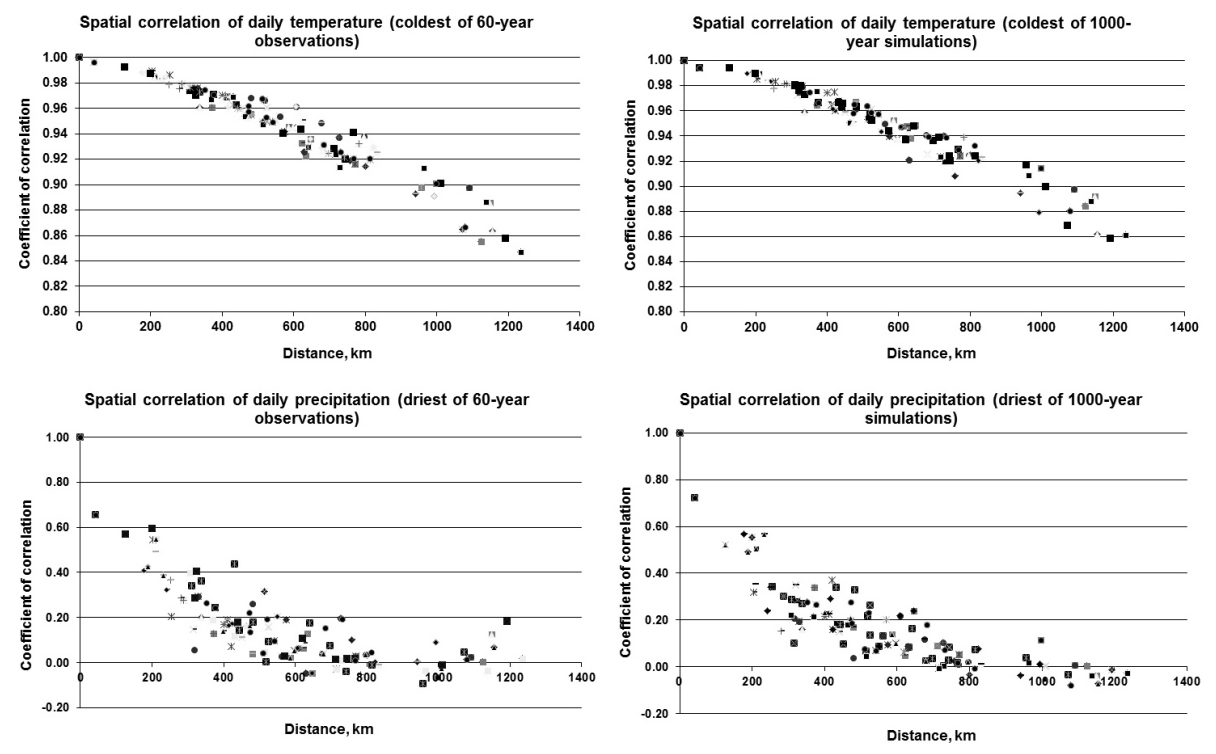

Figure 4. Spatial correlations of the observed (left) and generated (right) daily air temperature and precipitation in the study basin.

Table 1. Definition of States of Freshet and Low-Water Events.

\begin{tabular}{llll}
\hline Criterion & Description & Criterion & Description \\
\hline FI $\leq 0$ & No freshet event & LWI $\geq 0$ & No low-water event \\
$0<\mathrm{FI} \leq 1.0$ & Mild freshet event & $-1.0 \leq \mathrm{LWI}<0$ & Mild low-water event \\
$1.0<\mathrm{FI} \leq 1.5$ & Moderate freshet event & $-1.5 \leq \mathrm{LWI}<-1.0$ & Moderate low-water event \\
$1.5<\mathrm{FI} \leq 2.0$ & Severe freshet event & $-2.0 \leq \mathrm{LWI}<-1.5$ & Severe low-water event \\
$\mathrm{FI}>2.0$ & Extreme freshet event & $\mathrm{LWI}<-2.0$ & Extreme low-water event \\
\hline
\end{tabular}

the diagonal, and zero values for all entries off the diagonal. The off-diagonal elements give information about the specific nature of the forecast errors. To summarize the performance of multi-criteria forecast, we used two statistics: (1) forecast accuracy denoting overall fraction of the forecasts in the correct category; and (2) Heidke skill score showing the accuracy of the forecast in predicting the correct category, relative to that of random chance (Wilks, 1995).

As one can see from Table 2, accuracy of the freshet and low-water event forecasts are 0.590 and 0.544 , respectively, i.e. slightly more than half forecasted events fall in the same category as the observed ones. Heidke skill score equals $34.3 \%$ for freshet and $19.2 \%$ for low-water event, i.e. in both cases the forecasts give some additional information comparing with the random chance, but freshet event forecast is notably better than the forecast of low-water event.

It is easy to see from Table 2 that the forecast performance is improving with decrease of the number of categories. This result can be demonstrated, for example, by computing the above statistics for both observations and forecasts from $2 \times 2$ contingency table with only frequencies of "yes" and "no" ("yes"means that the event did occur or forecasted; "no" means that the event did not occur or not forecasted). Taking away all event categories listed in Table 2, except "yes-no", one can see that the freshet event forecast accuracy has increased to 0.783 whereas accuracy of the lowwater event forecast has increased to 0.656 (see Table 2). In other words the overall fraction of the forecasts in the correct category has risen by $10-20 \%$ depending on the event forecasted. Heidke skill score has also increased and became of $56.9 \%$ for freshet and $30.8 \%$ for low-water event forecast.

As an additional measure of relative skill of the ensemble forecast over that of climatology, we used Brier skill score (BSS) criterion common to meteorological probabilistic forecasting (Wilks, 1995). Essence of the BSS is close to that of the well-known hydrological Nash-Sutcliffe efficiency criterion: the former is the ratio of the mean squared error of the ensemble forecast probability to the mean squared error of the climatology forecast probability. We found that the number of successful forecasts was maximal if the events did not occur: in these cases BSS $=0.612$ for freshet-forecast and $\mathrm{BSS}=0.283$ for low-water-forecast. The worst forecasts were obtained for moderate freshet events $(B S S=0.262)$ and mild low-water events $(\mathrm{BSS}=0.088)$. Again, the forecast skill was found to be increased with decreasing number of categories. For binary (yes/no) event forecast BSS has reached $0.61-0.62$ for freshet event and $0.24-0.28$ for low- 
Table 2. Multi-Category Contingency Table of the Ensemble Event Forecasts (diagonal (bold) elements denote frequencies of the forecasted events of the specific category that meet the observed events in the same category).

Frequency of occurrence of freshet event

\begin{tabular}{|c|c|c|c|c|c|c|c|}
\hline & & \multicolumn{6}{|c|}{ Observed category } \\
\hline & & No event & Mild & Moderate & Severe & Extreme & Total \\
\hline \multirow{5}{*}{$\begin{array}{l}\text { Forecast } \\
\text { category }\end{array}$} & No event & 0.421 & 0.057 & 0.010 & 0.016 & 0.002 & 0.506 \\
\hline & Mild & 0.109 & 0.140 & 0.054 & 0.015 & 0.022 & 0.340 \\
\hline & Moderate & 0.015 & 0.028 & 0.028 & 0.003 & 0.005 & 0.079 \\
\hline & Severe & 0.007 & 0.014 & 0.022 & 0.000 & 0.004 & 0.047 \\
\hline & Extreme & 0.001 & 0.003 & 0.023 & 0.000 & 0.001 & 0.028 \\
\hline \multicolumn{2}{|c|}{ Total } & 0.553 & 0.242 & 0.137 & 0.034 & 0.034 & 1.000 \\
\hline \multicolumn{8}{|c|}{ Frequency of occurrence of low-water event } \\
\hline & & \multicolumn{6}{|c|}{ Observed category } \\
\hline & & No event & Mild & Moderate & Severe & Extreme & Total \\
\hline \multirow{5}{*}{$\begin{array}{l}\text { Forecast } \\
\text { category }\end{array}$} & No event & 0.281 & 0.143 & 0.000 & 0.000 & 0.000 & 0.424 \\
\hline & Mild & 0.184 & 0.263 & 0.000 & 0.000 & 0.000 & 0.447 \\
\hline & Moderate & 0.017 & 0.091 & 0.000 & 0.000 & 0.000 & 0.108 \\
\hline & Severe & 0.000 & 0.021 & 0.000 & 0.000 & 0.000 & 0.021 \\
\hline & Extreme & 0.000 & 0.000 & 0.000 & 0.000 & 0.000 & 0.000 \\
\hline \multicolumn{2}{|c|}{ Total } & 0.482 & 0.518 & 0.000 & 0.000 & 0.000 & 1.000 \\
\hline
\end{tabular}

water event. Importantly, for all categories forecast allowed obtaining additional information compared to climatology.

\section{Conclusions}

Despite the evidence advances in long-term (e.g. seasonal) forecasting methodology, forecasts remain far from perfect. Pathway, which could lead, in our opinion, to increase effectiveness of long-term hydrological forecast and satisfaction of rising society's demand for forecasts, lies in improving description of uncertainty of meteorological conditions for the forecast lead-time. In this paper, we propose ensemble forecasting technique, which combines the deterministic hydrological model with the spatially distributed stochastic weather generator. A hydrological model, which is forced by synthetic weather data, reproduces large ensemble of hydrological system responses to a lot of possible meteorological impacts, including those that did not recorded for the period of observations. Large-size ensemble can provide fresh opportunities for statistical interpretation of the forecasts and their use for water management. Indeed, the extent in which floods and droughts can be mitigated by management of water stored in reservoirs depends on capability of a forecasting method to give early outlook on magnitude and probability of occurrence of possible extreme hydrological event.

We applied the developed technique for 3-month-aheadforecasting of freshet/low-water seasonal inflow into large Cheboksary reservoir and verified the technique on the basis of the hindcast simulations for 29 spring and summer seasons beginning from 1982 (the year of the reservoir filling to capacity). The simulated large-size (500-hydrograph) ensemble of forecasts allowed us deriving reliable estimates of probability of possible hydrological event occurrence.

Acknowledgements. The research related to developing methods of ensemble forecast was financially supported by the Russian Foundation for Basic Researches (grant No. 13-05-00562). Part of the presented research related to developing forecast verification technique was financially supported by the Russian Ministry of Education and Science (grant No. 14.B25.31.0026). The present work has been carried out within the framework of the Panta Rhei Research Initiative of the International Association of Hydrological Sciences (IAHS).

\section{References}

Cloke, H. L. and Pappenberger, F.: Ensemble flood forecasting: a review, J. Hydrol., 375, 613-626, 2009.

Connelly, B., Braatz, D. T., Halquist, J. B., DeWeese, M., Larson, L., and Ingram, J. J.: Advanced hydrologic prediction system, J. Geophys. Res.-Atmos., 104, 19655-19660, 1999.

Gelfan, A. N. and Motovilov, Yu. G.: Long-term hydrological forecasting in cold regions: retrospect, current status and prospect, Geogr. Compass, 3, 1841-1864, 2009.

Gelfan, A. N. and Motovilov, Yu. G.: Developing methods of flood risk assessment and long-term flood forecasting on the basis of dynamic-stochastic model, in: Proceedings of the All-Russian 
Conference on River Runoff: Spatial-Temporal Variability and Hydrological Extremes, Moscow, Russia, November 2014, 145164, 2014 (in Russian).

Gelfan, A., Motovilov, Yu., Krylenko, I., Moreido, V., and Zakharova, E.: Testing robustness of the physically-based ECOMAG model with respect to changing conditions, Hydrol. Sci. J., doi:10.1080/02626667.2014.935780, in press, 2014.

Krzysztofowicz, R.: The case for probabilistic forecasting in hydrology, J. Hydrology, 249, 2-9, 2001.

Kuchment, L. S. and Gelfan, A. N.: Long-term probabilistic forecasting of snowmelt flood characteristics and the forecast uncertainty, in: proceedings of IAHS symposium on quantification and reduction of predictive uncertainty for sustainable water resources management, Perugia, Italy, July, 213-221, 2007.

Luo, L., Wood, E. F., and Pan, M.: Bayesian merging of multiple climate model forecasts for seasonal hydrological predictions, J. Geophys. Res., 112 D10102, doi:10.1029/2006JD007655, 2007.

McKee, T. B., Doeskin, N. J., ad Kleist, J.: The relationship of drought frequency and duration to time scales, in: Proceedings of the Eighth Conference on Applied Climatology, Anaheim, CA, 17-23 January, 179-184, 1993.
Motovilov, Yu. and Gelfan, A.: Assessing runoff sensitivity to climate change in the Arctic basin: empirical and modelling approaches, Cold and Mountain Region Hydrological Systems Under Climate Change: Towards Improved Projections, edited by: Gelfan, A., Yang, D., Gusev, E., and Kunstmann, H., IAHS Publications, 360, 105-112, 2013.

Motovilov, Yu. G., Gottschalk, L., Engeland, K., and Belokurov, A.: ECOMAG - regional model of hydrological cycle. Application to the NOPEX region, Department of Geophysics, University of Oslo, Institute Report Series no. 105, p. 88, 1999a.

Motovilov, Yu., Gottschalk, L., Engeland, K., and Rodhe, A.: Validation of a distributed hydrological model against spatial observation, Agr. Forest Meteorol., 98-99, 257-277, 1999b.

Nalbantis, I.: Evaluation of a hydrological drought index, Eur. Water, 23/24, 67-77, 2008.

Svanidze, G. G.: Mathematical Modeling of Hydrologic Series. Water Resources Publications, Fort Collins, Colorado, USA, 1980.

Wilks, D. S.: Forecast Verification: Statistical Methods in the Atmospheric Sciences, Academic Press, New York, USA, 1995. 